University of San Diego

Digital USD

Spring 5-25-2019

\title{
Optimizing an Order Set to Streamline Health Care Delivery in the Emergency Department for Patients Requiring Hiv Post-Exposure Care
}

Marlisha Smith

University of San Diego, msmith108@gmail.com

Follow this and additional works at: https://digital.sandiego.edu/dnp

Part of the Nursing Commons, Pharmacy and Pharmaceutical Sciences Commons, and the Public Health Commons

\section{Digital USD Citation}

Smith, Marlisha, "Optimizing an Order Set to Streamline Health Care Delivery in the Emergency Department for Patients Requiring Hiv Post-Exposure Care" (2019). Doctor of Nursing Practice Final Manuscripts. 100.

https://digital.sandiego.edu/dnp/100

This Doctor of Nursing Practice Final Manuscript is brought to you for free and open access by the Theses and Dissertations at Digital USD. It has been accepted for inclusion in Doctor of Nursing Practice Final Manuscripts by an authorized administrator of Digital USD. For more information, please contact digital@sandiego.edu. 


\section{OPTIMIZING AN ORDER SET TO STREAMLINE HEALTH CARE DELIVERY IN THE EMERGENCY DEPARTMENT FOR PATIENTS REQUIRING HIV POST- EXPOSURE CARE}

UNIVERSITY OF SAN DIEGO

Hahn School of Nursing and Health Science

Beyster Institute for Nursing Research

\section{DOCTOR OF NURSING PRACTICE PORTFOLIO}

by

Marlisha Smith

A portfolio presented to the

FACULTY OF THE HAHN SCHOOL OF NURSING AND HEALTH SCIENCE:

UNIVERSITY OF SAN DIEGO

In partial fulfillment of the

requirements for the degree

DOCTOR OF NURSING PRACTICE

May 2019 
Optimizing an Order Set to Streamline Health Care Delivery in the Emergency

Department for Patients Requiring HIV Post-Exposure Care

Marlisha Smith

University of San Diego

Hahn School of Nursing 


\begin{abstract}
Background: The Centers for Disease Control and Prevention (CDC) updated guidelines to support efforts to decrease the number of Human Immunodeficiency Virus (HIV) incidences. The principle method for risk reduction utilized in the guidelines is the 28 day non-occupational post-exposure prophylaxis (nPEP) antiretroviral course administered within 72 hours of exposure. Despite this recommendation, adherence to a full 28-day course nPEP is very low nationally. Root cause analysis completed at the study site indicated that the use of an outdated order set caused unwarranted variability in care which accounted for failure in the delivery of appropriate nPEP care and low medication adherence. Order sets are clinical standard tools within computerized provider order entry systems to reduce unwanted variation and improve care processes.

Purpose: The purpose of this quality improvement project was to standardize and optimize patient care in accordance with current clinical guidelines through the use of an updated order set. The multidisciplinary approach to quality improvement is viable to ensuring accurate and appropriate evaluation, treatment, follow-up, and prevention of HIV in patients seen in the Emergency Department after an exposure.

Evidence-based Intervention: A team of interprofessional experts developed an updated evidence-based nPEP order set that follows current CDC clinical practice guidelines. Implication for Practice: This project aligns the Emergency Department's nPEP protocol with current best practice and clinical guidelines.
\end{abstract}

Keywords: Nonoccupational exposure, nPEP, sexual assault, HIV, emergency room 
Optimizing an Order Set to Streamline Health Care Delivery in the Emergency Department for Patients Requiring HIV Post-Exposure Care

\section{Introduction}

The Human Immunodeficiency Virus (HIV) is a major medical crisis in the United States, with new cases occurring daily. Today, more than 1.2 million people are living with HIV in the United States. About 1 in 7 of those infected are unaware of their infection, thus increasing the risks for other to contract HIV (CDC, 2019). Despite the massive decline in HIV transmission over the years, the epidemic continues to be a public health issue. The decrease in annual HIV infections and diagnoses can be attributed to prevention strategies and services. In 2010, former President Barack Obama created a comprehensive National HIV/AIDS Strategy that concentrated on four core measures: reducing new infections, increasing access to care and improving health outcomes for people living with HIV, reducing HIV-related disparities and health inequities, and achieving a coordinated National response to the epidemic (Bradley-Springer, 2015). Another coexisting governmental initiative, Healthy People 2020, confirms that HIV is a major crisis. The objective remains to prevent HIV infection, related illnesses, and deaths (United States Department of Health and Human Services [HHS], Healthy People 2020, 2018). The initiative Getting to Zero, aims to end the HIV epidemic through HIV prevention programs, testing, treatment, and supporting activities. Getting to Zero supports increased awareness of HIV through engaging health care systems. This is done through implementing pre-exposure and post-exposure prophylaxis interventions, using data to improve outcomes, and addressing disproportionalities in HIV related health care 
(County of San Diego Health and Human Services Agency Public Health Services, 2017).

\section{Background}

Non-occupational exposure to HIV is defined as an isolated exposure outside of a health care setting to blood, genital secretions, or other potentially infectious body fluids that may contain the virus. Individuals who are involved in high-risk activities including sexual assault, consensual sexual engagement, and/or intravenous drug use are at risk for acquiring HIV. In 2017, non-occupational exposure to HIV accounted for $99.4 \%$ of new HIV diagnoses in the United States and 6 dependent areas (CDC, 2019). In 2016, The CDC updated the Guidelines for antiretroviral post-exposure prophylaxis after sexual, injection drug use, or non-occupational exposure to HIV. These clinical practice guidelines were published as a national protocol to guide care of patients requiring nPEP.

\section{HIV Post-exposure Management}

Exposure to HIV is considered a medical emergency. In their clinical practice guidelines, the CDC summarized recommendations for management and treatment of patients who present to the Emergency Department (ED) with substantial risk for HIV acquisition. Evidence-based clinical practice guidelines are specific practice recommendations that have been derived from a methodologically rigorous review of the best evidence on a specific topic (Melnyk, 2018). Clinical care outlined in this guideline include: a comprehensive history and physical examination, testing and prophylactic treatment of sexually transmitted infections (STIs), follow-up care, and counseling after ED discharge. Non-occupational post-exposure prophylaxis (nPEP) is the recommended 28-day antiretroviral regimen used to reduce the risk of HIV transmission. 
After a known or suspected exposure to HIV, patients should seek immediate treatment, with one option being in the ED. This has caused ED providers to play a major role in the risk reduction of HIV. The CDC states that within 72 hours post-exposure, an initial evaluation should be conducted by a provider to rapidly identify, evaluate, and treat. Labs that are clinically indicated should be ordered and drawn. The first dose of recommended antiretrovirals along with other STI prophylaxis should be initiated in the ED.

At ED discharge, a 5-day starter pack of antiretroviral medication is prescribed as a bridge until patients are seen for follow-up. A seamless transition of care for follow-up is invaluable for patients. It allows patients to receive the remaining 23-day supply of antiretrovirals, appropriate screening, counseling, and follow-up testing for HIV and other infections. When the guidelines are followed correctly by providers and patients, the use of nPEP can stop HIV transmission decreasing the risk of infection. Adherence to the recommended 28-day treatment without interruption is essential. The efficacy of nPEP is closely correlated with the compliance of the medication regimen (Oldenburg, Bärnighausen, Harling, Mimiaga, \& Mayer, 2013). Research suggests that nPEP effectiveness is higher than $80 \%$ when used as prescribed. Despite these numbers and having a comprehensive National strategy for the prevention of HIV in place, the adherence rates of nPEP therapy remains unquestionably low based on evidence throughout the years. Per the CDC, the adherence rate to nPEP ranges from $29 \%$ to $71 \%$. Available research suggests that care provided to patients post HIV exposure in the ED is often suboptimal. Merchant et al. (2008) found that patients who presented to the ED did 
not consistently receive all recommended testing and prophylaxes based on clinical recommendations, attributing to variability in clinician practice.

Gaps in care of patients requiring nPEP can be a result of outdated order sets that cause variation from current best practice. Discrepancies between recommended best practice and care provided in the ED can be seen in the areas of: inappropriate laboratory ordering, medication prescribing errors, and lack of rapid access to care for follow-up after ED discharge (Schilling et al., 2017). The aforementioned discrepancies increase the risk of significant adverse outcomes; the most significant being the transmission of HIV in this patient population. Therefore, accurate and appropriate management of patients after exposure to HIV is paramount. Failure to receive care that meets standards based on clinical guidelines and national recommendations, warrants quality improvement evaluation and intervention (Schilling et al., 2017).

\section{Problem Statement}

Observational analysis identified that management and treatment provided in a Southern California ED did not incorporate current evidence contributing to substantial gaps in care. Variations in health care delivered to patients after an exposure to HIV were noted in the areas of prescribing nPEP, ordering laboratory tests, and also the failure in linkage to clinic care. These discrepancies were a result of not updating the Southern California ED's electronic health record order set following the 2016 changes to the CDC's clinical guidelines for nPEP. When national guidelines were updated, the site failed to make applicable changes to the order set causing care to digress from national standards. The aim of this quality improvement project was to standardize and optimize patient care in accordance with current clinical guidelines by updating the nPEP order set. 


\section{Review of the Literature}

Research indicates that the use of electronic order sets led to better standardized care for patients seen post-exposure to HIV. Grissinger (2014) explained that the use of standardized orders decreased incorrect or incomplete prescribing, systematized patient care, and guaranteed clarity when communicating medical tasks. Another organization that supports standardized and consistent evidence-based approaches to nPEP management is the California Department of Public Health. Implementing standardized measures in the ED improves delivery, efficiency, and safety of health care. A large meta-analysis completed by Mayorgora in 2013 supports the effectiveness of standardized order sets for optimizing evidence-based medical care, finding that order sets improved adherence and timelines of administration of recommended therapies for patients. The systematic approach of order sets create uniformity amongst experts and decreases inconsistencies in nPEP care based on current consensus guidelines. Without the use of evidence-based order sets, care provided in the ED to patients with nonoccupational HIV exposure often does not reflect National policies and procedures.

Using a survey, Bamberger (2003) identified that ED providers offered HIV postexposure prophylaxis less often after consensual exposures than sexual assault or occupational exposures. He identified that this was due to unfamiliarity with the prophylactic regimen causing providers to be uncomfortable prescribing nPEP. Stratification of prescribing HIV post-exposure prophylaxis is inversely proportional to the numbers of incidence of HIV caused by this exposure type in the United States (Stanley et al., 2017). This may significantly impact the clinical outcomes of patients after an exposure. As technology advances, it is important to put systems in place to 
assist providers in providing evidenced-based care for patients after an HIV exposure and to prevent medical errors associated with nPEP care. Order sets serve as templates to guide appropriate evidence-based care. Emergency Department providers play a critical role in utilizing the nPEP order set because accurate and timely orders are critical when providing effective HIV post-exposure prophylaxis. A recent retrospective study conducted by Guo et al. (2015), revealed that customized order sets decreased error rates from $38 \%(n=723)$ to $24.8 \%(n=661)(G u o$, Chung, Wiess, Veltri, \& Minamoto, 2015).

Similarly, Britton et al. (2013) conducted a retrospective review to evaluate the implementation of an evidence-based order set that was consistent with the CDC guidelines for non-occupational exposures. The study compared the rate of provider compliance with the CDC guidelines before $(n=322)$ and after $(n=131)$ implementation of the order set. Results revealed a statistically significant increase in compliance $(\mathrm{p}<0.001)$ of the clinical recommendations. Computerized order sets for the management and treatment of patients with non-occupational exposure to HIV significantly improved ED providers' compliance with CDC treatment guidelines.

Quality improvement studies evaluating the use of computerized provider order entry and clinical decision support systems have demonstrated that implementing order sets improved adherence to recommended treatment protocols, thereby reducing variation in care. Order sets minimize medical errors, improve speed, and accuracy of care provided in the ED. HIV post-exposure care is complex and requires the utilization of many ED resources. Employing order sets minimizes deviations from clinical guidelines and applicably utilizes hospital resources. Evidence confirms that guideline-based order sets decreased prescribing errors, increased the used of best clinical practice, and 
improved the consistency of care provided to patients with non-occupational exposure to HIV seen in the ED (Britton, Bloch, Strout, \& Baumann, 2013).

Although order sets have been shown to support evidence-based standardized care, when they are not adequately maintained they are the sources of gaps, variations, and outdated clinical practices. Order sets require regular evaluation and maintenance to meet the ever-changing needs of today's practice. Medication and other orders within the order set need to be revised frequently based on changes in evidence, supply, and processes (Britton, Bloch, Strout, \& Baumann, 2013). Order set require periodic reviews as evidence and care processes change to reinforce best practice and eliminate outdated practice.

\section{Methods}

\section{Organizational "Gap" Analysis of Project Site}

A retrospective review of data obtained through chart review revealed the utilization of an outdated order set. The existing order set did not reflect current evidence available for HIV post-exposure care causing discordance between clinical practice and the CDC clinic guidelines. Hence, creating inefficient care to be provided in the ED setting.

\section{Purpose}

The purpose of this quality improvement project was to standardize and optimize patient care in accordance with current clinical guidelines through the use of an updated order set. A team of interprofessional experts developed an updated evidence-based nPEP order set based on current CDC clinical practice guidelines. This was done in effort to 
streamline health care delivery of those requiring expediated care after known or suspected exposure to HIV.

\section{Setting and Sample}

The setting of the project was a Southern California acute care setting. The study site houses an ED, a level I Trauma Center, and an HIV specialty clinic. The project involved both the site's ED and HIV specialty clinic. The gaps in care were siteidentified. A retrospective review of data obtained through chart review for patients seen for non-occupational exposure to HIV was completed between January 1, 2017 and March 8, 2018. The order set used to guide care during this timeframe was identified as not up-to-date in regards to $\mathrm{CDC}$ recommendations and required maintenance to provide accurate standard of care.

\section{Ethical Issues}

Institutional review board approval was obtained from the academic's and institution's review boards prior to the initiation of the practice project. All data collected as part of this project were cleansed of patient identifiers.

\section{Quality Improvement Team}

In 2017, a multidisciplinary committee comprised the author, a Transitions of Care nurse practitioner, an ED physician, Infectious Disease physicians, clinical pharmacists, and Epic clinical informatics analysts convened to complete a root cause analysis. The gaps in the medical management of patients presenting to the ED with nonoccupational HIV exposure included loss to follow-up with the HIV specialty clinic. Monthly meetings took place to discuss strategies to improve care. The team decided that the area of change would be the nPEP order set. The team decided to build a new order 
set that conformed to CDC evidence-based clinical practice guidelines and current formulary.

\section{Evidence-Based Intervention}

Optimization of the existing nPEP order set. A multidisciplinary approach was taken to build a new order set to streamline the health care delivery of patients requiring nPEP. The process change emphasized specific quality and safety improvements. Focus was placed on developing a tool that directly addressed the team's organizational goals. An evidence review was completed to ensure relevance of change. The existing order set was used as a framework but was modified to comply with current evidence and standards. Clinical experts provided input and oversight for all content changes made. Design revisions to the clinical pathway schematics was completed to better guide providers with nPEP initial and follow-up care.

nPEP order set modifications. Exposure-specific orders which allowed the separation of occupational exposures from non-occupational exposures. Occupational exposure to HIV differs from non-occupational in that the exposure occurs in the workplace. Differentiating this type of exposure was a critical change in the project. It allowed for the method of exposure to be more readily identified. Possible exposure to HIV in an occupational setting does not involve sexual contact, therefore the STI labs are not needed. In light of rising health care costs, removing STI screenings from the occupational exposure order set eliminated hospital waste by utilizing more specific and efficient ordering practices. This change also allowed for appropriate collection of HIV exposure-type. 
Additionally, sex-specific orders (male vs. female), were implemented. Sexspecific orders also help to reduce health care waste by removing unwarranted lab tests. Male patients do not require evaluation testing for vaginosis or pregnancy. Removing these labs for male patients decreased inappropriate ordering of laboratory tests while maintaining quality of care.

More importantly, sex-specific changes were made because the nPEP formulary had changed for women of child-bearing age. The initial nPEP treatment for male and female in the project setting included: Tenofovir and emtricitabine (Truvada) plus dolutegravir (Tivicay). However, in May 2018, the CDC published a warning statement regarding potential fetal harm and neural tube defects surrounding the use of Dolutegravir. This prompted the transition to twice daily Raltegravir (Isentress) for use in female patients requiring nPEP. Raltegravir has been seen to have the same efficacy as Dolutegravir in the female population in previous studies conducted. This project removed Dolutegravir from the nPEP regimen for women in order to comply with current practice and reduce adverse events. Confirming that medications matched the hospital drug formulary and were correctly dosed was essential in the development and maintenance of an order set (Leu, Morelli, Chung, \& Radford, 2013).

Lastly, an electronic referral, InBasket, to the hospital's HIV specialty clinic was implemented. InBasket notified the HIV Transition of Care Specialist that a patient had been seen in the ED for non-occupational post-exposure and required a follow-up visit within 3-5 days of being seen in the ED. This change was important because the patient only receives a 5-day supply of nPEP medication upon ED discharge. Follow-up is necessary in order for the patient to receive the remaining dosage of the medication. Also, 
additional counseling and monitoring is provided at the follow-up visits. For ED providers and specialists, electronic referral is an invaluable form of communication. This component of the order set was added to ensure a safe transition of patient care among providers and improve the referral process. It also provided a mechanism to track referrals.

All changes made within the order set were done to integrate and coordinate care. The new order set ensures appropriate care delivery and satisfies recommended practice guidelines.

Education of health care team. An email providing education on the new updated order set and key practice recommendations was sent to ED providers and HIV specialty clinic providers. Ockene and Zapka (2000) believed that education focusing on the provider was a major method used to affect patient care and improve outcomes. Boaz, Baeza, and Fraser (2011) also supported provider education and identified that a multifaceted approach promotes effective implementation of research into practice.

\section{Results}

Invaluable improvements in health care delivery of post-exposure care can be achieved through the implementation of an updated evidence-based order set in the ED. By utilizing this quality improvement method to standardize the use of current therapy, order recommended laboratory tests, and ensure prompt follow-up care in an HIV specialty clinic, this project works to reduce errors and improve quality of health care delivery in the ED. This order set aligns nPEP care with current best practice and clinical guidelines. 


\section{Limitations}

As with other studies, there were limitations to reflect upon. One limitation was that the data collected was obtained from one ED of the entire site system. Although this project is reproducible, the data obtained does not reflect nPEP order sets of other EDs in the site system. Also, throughout the course of the project several challenges were met. One of the most difficult tasks was scheduling meetings that coincide with each team members' schedule. Implementing change in a large system entailed going through multiple channels to obtain approval. This was very time consuming and tedious which placed multiple delays on the project. The build out of the new order set was also very laborious due to the robust workflow processes and order set components.

Another major limitation of the study was the lack of ongoing communication between the two speciality areas involved in this project: the ED and the HIV speciality clinic. Despite keeping an open line of communication with each department representative, there were times when information was not dispersed among other key team members. As a result, another order set for Bloodborne Pathogen Exposure was being updated and revised at the same time. Since HIV is a bloodborne pathogen, some hospital officials did not see the need for the new nPEP order set placing a delay on production.

\section{Discussion}

Evidence-based practice is encouraged throughout literature, however, there is confirmation that obsolete order sets used in any setting can deviate care from best clinical practice. The use of outdated order sets contributes to serious errors in diagnosis and treatment. Inadequate evaluation and maintenance of order sets lead to variation in 
care causing outdated practice. The aim of this project was to improve care processes to follow clear CDC consensus guidelines for care of patients requiring nPEP. The design revisions including default orders support a reduction in errors. In addition to the decreases in incongruencies in care and variations in treatment, this order set increases the utilization of best clinical evidence by ED providers. This project is equally beneficial for patient and providers alike. The patients are ensured improved quality of care delivery during a time of crisis. Provider workflow is efficient and concise, which improves referrals and linkage to care promoting collaboration amongst providers of different specialties.

\section{Conclusion}

Emergency providers oversee the majority of exposures to HIV that prompt request for post-exposure prophylaxis (Stanley et al., 2017). This project implemented multiple steps within an order set to streamline health care delivery. By optimizing the nPEP order set to guide the care of patients after a non-occupational exposure to HIV, the management and treatment adheres to CDC guidelines. The enhanced order set is a method that decreases prescribing errors, increases the use of evidence-based clinical practice, and streamlines care delivery to patients after a non-occupational HIV exposure. Giving ED providers this proper tool needed to care for these patients will promote appropriate treatment and prevent dangerous adverse effects.

Moreover, the order set encourages nPEP follow-up with a provider, specifically an HIV specialist. This component is needed to improve rates of follow-up and completion of nPEP to reduce the risk of acquisition. Follow-up appointments are imperative to the continuation of the medication regimen, screenings of other communicable conditions, 
and counseling. The expectation is that all patients who are HIV negative for nPEP, that come into the ED will have the best chance of remaining negative with the correct use of the nPEP protocol by providers and compliance from patients.

The optimized order set is a method to bridge gaps in care of patients exposed to HIV non-occupationally in the ED. The changes made are sustainable additions to the electronic medical record. However, success relies heavily on ongoing research, frequent evaluations, and updates to the order set to remain optimally relevant. 


\section{References}

Boaz, A., Baeza, J. \& Fraser, A. (2011). Effective implementation of research into practice: an overview of systematic reviews of the health literature. $B M C$ Research Notes, 4(1), 212. doi:10.1186/1756-0500-4-212

Bradley-Springer, L. (2015). The National HIV/AIDS Strategy: Updated for the future. Journal of the Association of Nurses in AIDS Care, 26(6), pp.689-692.

Britton, D. J., Bloch, R. B., Strout, T. D., \& Baumann, M. R. (2013). Impact of a Computerized Order Set on Adherence to Centers for Disease Control Guidelines for the Treatment of Victims of Sexual Assault. The Journal of Emergency Medicine,44, 528-535. doi:10.1016/j.jemermed.2012.06.016

Centers for Disease Control (2016). Management of possible sexual, injecting-drug-use, or other nonoccupational exposure to HIV, including considerations related to antiretroviral therapy: Public health service statement. PsycEXTRA Dataset. doi:10.1037/e547622006-001

Center for Disease Control and Prevention (2018). HIV/AIDS: Programs. Retrieved from: http://www.cdc.gov/hiv/prevention/programs/

Centers for Disease Control. (2019). U.S. Statistics. Retrieved from https://www.hiv.gov/hiv-basics/overview/data-and-trends/statistics

Chacko, L., Ford, N., Sbaiti, M., \& Siddiqui, R. (2012). Adherence to HIV post-exposure prophylaxis in victims of sexual assault: a systematic review and meta-analysis. Sexually Transmitted Infections, 88(5), 335-341.County of San Diego Health and Human Services Agency Public Health Services. (2016, April 27). HIV/AIDS Epidemiology Report 2015. Retrieved from 
https://www.sandiegocounty.gov/content/dam/sdc/hhsa/programs/phs/documents/ HIV_Epi_Report_2015_FINAL.PDF

County of San Diego Health and Human Services Agency Public Health Services. (2017, July 26). Getting to Zero. Retrieved from https://www.sandiegocounty.gov/hhsa/programs/phs/hiv_std_hepatitis_branch/Ge tting_to_Zero.html

Grissinger, M. (2014). Guidelines for standard order sets. P \& T: A Peer-Reviewed Journal for Formulary Management, 39, 10-11.

Guo, Y., Chung, P., Weiss, C., Veltri, K., \& Minamoto, G. Y. (2015). Customized orderentry sets can prevent antiretroviral prescribing errors: a novel opportunity for antimicrobial stewardship. P \& T: A Peer-Reviewed Journal for Formulary Management, 40, 353-360.

Idemoto, L., Williams, B., \& Blackmore, C. (2016). Using lean methodology to improve efficiency of electronic order set maintenance in the hospital. BMJ Quality Improvement Reports, 5(1). doi:10.1136/bmjquality.u211725.w4724

Melnyk, B. M., \& Fineout-Overholt, E. (2018). Evidence-based practice in nursing \& healthcare: A guide to best practice. Philadelphia, PA: Lippincott Williams \& Wilkins.

Merchant, R. C., Kelly, E. T., Mayer, K. H., Becker, B. M., Duffy, S. J., \& Pugatch, D. L. (2008). Compliance in Rhode Island emergency departments with American Academy of Pediatrics Recommendations for Adolescent Sexual Assaults. Pediatrics, 121(6). doi:10.1542/peds.2007-3100 
Ockene, J.K., \& Zapka, J.G. (2000). Provider education to promote implementation of clinical practice guidelines. Chest, 118(2). doi:10.1378/chest.118.2_suppl.33s

Oldenburg, C. E., Bärnighausen, T., Harling, G., Mimiaga, M. J., \& Mayer, K. H. (2013). Adherence to Post-Exposure Prophylaxis for Non-forcible Sexual Exposure to HIV: A Systematic Review and Meta-Analysis. AIDS and Behavior, 18, 217-225. doi:10.1007/s10461-013-0567-0

Schilling, S., Deutsch, S. A., Gieseker, R., Molnar, J., Lavelle, J. M., \& Scribano, P. V. (2017). Improving HIV post-exposure prophylaxis rates after pediatric acute sexual assault. Child Abuse \& Neglect, 69, 106-115.

doi:10.1016/j.chiabu.2017.04.014

Stanley, K., Lora, M., Merjavy, S., Chang, J., Arora, S., Menchine, M., \& Jacobson, K. R. (2017). HIV Prevention and Treatment: The Evolving Role of the Emergency Department. Annals of Emergency Medicine, 70(4). doi:10.1016/j.annemergmed.2017.01.018

United States Department of Health and Human Services, Healthy People 2020. (2018, March 6) HIV: Overview. Retrieved from https://www.healthypeople.gov/2020/topics-objectives/topic/HIV?topicid 